\title{
Prison Staff and Family Visits: United Kingdom Case Study
}

\author{
Avon Hart-Johnson ${ }^{1, ~ *, ~ G e o f f r e y ~ J o h n s o n ~}{ }^{2}$ \\ ${ }^{1}$ College of Social and Behavioural Sciences, Walden University, Minneapolis, MN, United States \\ ${ }^{2}$ DC Project Connect (DCPC) Independent Research, DCPC, Washington, DC, United States
}

Email address:

avon.hart-johnson2@mail.waldenu.edu (A. Hart-Johnson), geoffreyalanjohnson@gmail.com (G. Johnson)

${ }^{*}$ Corresponding author

\section{To cite this article:}

Avon Hart-Johnson, Geoffrey Johnson. Prison Staff and Family Visits: United Kingdom Case Study. Humanities and Social Sciences. Vol. 8, No. 2, 2020, pp. 63-72. doi: 10.11648/j.hss.20200802.13

Received: April 19, 2020; Accepted: May 13, 2020; Published: May 27, 2020

\begin{abstract}
Recent studies have illuminated how families are affected by prison staff engagement during prison visits. Prison staff, by virtue of their positions, exercise power and authority over families during prison visits processes and potentially play a vital role in shaping inmate-family experiences. Although some interactions with prison staff may be cordial, others have been reported as stressful for visitors, especially families with children. Yet many family visitors endure stressful encounters since visits can serve as portals for bonding between inmates and their families. Over the past two decades, some prisons in the United Kingdom (UK) have invested in developing family-oriented practices and visits protocol, where prison staff oversee the visits process. This is in contrast to prison staff who tend to take on a police-officer mentality whereby policing behaviors or personal philosophies are aligned with implied suspicion, mistrust, and apprehension towards inmates and families. Given such, prison staff interpretation of their roles and engagement with families is of research interest. The purpose of this qualitative multiple case study was to understand how UK prison staff interpreted their roles during family visits and determine why this evolution towards family-focus visiting took place. To answer the research inquiry, a heterogeneous sample of prison staff from private and public sector prisons and advocacy center contractors employed at two prisons in the United Kingdom were recruited to provide broad perspectives on staff roles during the process of the visit. Within these facilities, we interviewed a total of $13(n=13)$ staff members. Data collection and semi-structured interviews occurred at two prison facilities and two advocacy centers. Three researchers conducted cross-case analyses and applied triangulation methods to establish a clear chain of evidence documented through step-by-step processes: audit trails; flexible and parallel data analysis during data collection, and first- and second-order coding processes. Findings from this study indicate that prison staff perceives their roles during the visiting processes as an evolution from traditional corrections-based punitive practices (i.e. policing) to a visiting program capable of accommodating three goals: low-stress family visits, inmate conformance, and reduced recidivism. This study adds to the literature on detailing how prison staff dichotomous roles were interpreted as balancing tensions between institutional controls and minimizing traumatic experiences for visiting families through prison-based interventions.
\end{abstract}

Keywords: Family Systems and Prison Staff, Family Prison Visits, Prison Staff Roles, Prison Staff, Family Engagement, Prison Visits, Multiple Case Study, United Kingdom Prison Visiting Process

\section{Introduction}

Recent studies have illuminated how families are affected by prison staff engagement during prison visits $[1,2]$. Prison staff, by virtue of their positions, exercise power and authority over families during prison visits and potentially play a vital role in shaping inmate-family experiences [2, 3]. Although some interactions with prison staff may be cordial, others have been reported as stressful for visitors, especially for families with children [3]. Many visitors endure stressful encounters since visits can serve as portals for bonding between inmates and their families $[5,6]$.

In many prisons located in the United States, draconian, retributive practices often govern how families are treated during prison visits [7]. In these settings, prison staff tend to follow harsh command and control protocols to prevent contraband, inmate infractions, and unlawful activities $[4,8$, 9]. Additionally, prison visiting policy and regulations can be 
subject to staff interpretation and vary by jurisdiction [3]. How staff interpret their roles when intersecting with families during the visits can provide insights on how to develop meaningful and less stressful visits programs for families. The literature is clear that prison visits can have multiple benefits, including a reduction in tension in visiting areas, a decline in institution misconduct, and ultimately, a reduction in recidivism [10]. In this study, we were specifically interested in prison staff behaviors as instituted during the visits programs. To address our research inquiry, we broadened our search to identify prisons with progressive models in the United Kingdom, one of several countries where family-centric programs exist [11].

\subsection{United Kingdom Family-Oriented Prisons}

Over the past two decades, some prisons in the United Kingdom (UK) have invested in developing family-oriented visiting practices and visits protocol [11]. Moreover, during 2011, the UK Ministry of Justice issued a mandate for all prisons to provide purpose-built visitor facilities, specifically for families [11]. In these progressive prisons, prison staff appear to be the arbiters who interpret and apply family-centric practices and protocols [12-15]. However, there are significant implementation differences in how prison staff carry out and enact their roles within UK penal institutions [16]. The purpose of this qualitative multiple case study was to understand how prison staff interpreted their roles during family visits and to determine why this evolution towards family-focus visiting took place.

\subsection{Family Systems Theory}

Family systems theory has been used to understand how families thrive after the onset of a crisis, such as family member incarceration, e.g. [5]. This theory is used to explore how the prison staff's roles and engagement in visiting settings might affect the family unit. Family systems operate on the alignment of three major concepts [17-18]: (1) family systems are interconnected, (2) the whole family unit is more than the sum of its parts, and (3) family systems have boundaries. According to the theoretical framework of Bowen [18], in the face of crisis, and in a state of disorganization, a family unit (e.g., immediate family in a household) will strive to regain congruence.

Arditti [5] maintained that continuous family relations through prison visits can provide stability in a time of distress during a family member's incarceration. Critical interconnections between families and inmates can occur through bonding during visits (e.g., child-parent contact during prison visits [17]).

\section{Literature Review}

During prison visits, family member displays of physical agitation, psychological distress, and panic can result from the actual or anticipated encounters with prison staff [7]. Visiting family members, while not incarcerated themselves, are sometimes treated by prison staff in similar punitive fashion as the inmates [19]. Hulley [14] suggested that some prison staff tend to take on a police-officer mentality, whereby policing behaviors or personal philosophies are aligned with implied suspicion, mistrust, and apprehension towards inmates and families. How prison staff view their roles may influence how they treat families, such as using a sensitive, retributive, or even a utilitarian approach to families and the inmates. A retributive approach might entail how inmates are adversely treated as justifiable because the punishment fits the crime [15]. A staff member who views their role as underpinned by a utilitarian philosophy might provide a lenient and sensitive approach to families [15]. Souryal [20] suggested that the authority and power asserted by staff can wield tremendous control over those who are under their command, including visiting families. However, the harsh or insensitive treatment of visitors by staff may not always be the norm, and inconsistencies exist.

In the United Kingdom, some prisons have implemented family-friendly programming for visitors. As indicated, prison staff who extend sensitivity towards visiting family members have observed multiple benefits in prison environments [10]. Buford [12] found that when prison staff showed common courtesy towards family and children, this can serve as a rehabilitative outcome for inmates. For example, prison visits from family can reduce inmate aggression [21]. Sudom and colleagues [22] suggested that family-based visits help the prison staff to experience less stressful work environments. Given these benefits, some prisons have adopted a holistic approach to reducing the adverse impact that carceral environments have on visiting families [23]. For instance, some facilities provide reception areas for visitor intake and welcome centers (also referred to as visitor centers) [10, 7]. These trends may signal a change in how prison staff view their roles and responsibility as they intersect with families in visiting environments.

Boudin [3] reported that prison visiting protocols and interior design are also important to the family experience and can vary by prison (i.e. public sector versus private sector prisons). Although research has been conducted from the perspective of what constitutes a good prison visit for families $[7,9,24]$, the literature lacks perspectives on how prison staff interpret their roles in family-centric visiting environments. The nature of these unanswered questions calls for a methodology that offers breadth and depth in understanding what Yin [25] offers as the how and why things occur.

\section{Methodology}

An exploratory, interpretive, multiple case study approach [25] was selected as the best method to address the research inquiry. Case studies are ideal for collecting rich and specific data, bound by context as gathered from participants in natural settings [26]. Interpretive case studies are used to understand the social world as constructed through the subjectivity of the research participants [27]. In this study, we describe data from qualitative interviews that were purposefully and 
systematically collected with the goal of understanding the prison staff's interpretation of their roles. Prison staff was considered: individuals who worked in prison visits halls or adjacent visiting centers (facilities dedicated to assisting visitors). Our initial research questions (RQs) were developed, then refined as we better understood the context of the research problem (see [30]). RQ1. How do prison staff interpret their roles during family visiting experiences? RQ2. How do prison staff use spatial areas, including their form, size, scale, and color, to represent family-visits spaces? RQ3. How do prison staff perceive the evolution of family-centric prison visiting environments?

\subsection{Sample Characteristics}

Our sample comprised two United Kingdom purposively selected prisons and two advocacy center central offices. To select the prisons, we conducted an examination of archival records, pamphlets, public service announcements, websites, public policy documents, and public and private prison websites. We selected two prisons that were identified as family-friendly visiting programs (i.e. catered to families, had play areas, and were noted for their family-based programs). In this qualitative study, a "case" is considered one of three groupings of prison workers. Private sector prison staff comprised the first case. Public sector prison staff were considered the second case. The third case comprised advocacy staff who had separate home offices but worked at one of the two prisons. This heterogeneous sample provided broad perspectives on prison staff roles. Each case constituted a unit of analysis. Within these facilities, we interviewed a total of $13(n=13)$ staff members. These cases included staff with varying titles, such as corrections officers, family contact staff, advocacy workers, volunteers, researchers, and managers/ supervisors. To protect the confidentiality of the participants, we do not disclose the numbers of respondents per group and we refer simply to the cases when describing results as public or private sector prisons or advocacy groups.

\subsection{Recruitment of Participants in the United Kingdom}

The research team originated in the United States. To proceed with our study, we first received approval from multiple ethics and governance institutions, including a United States university Institution Review Board, Her Majesty's Prison Service (UK), and the Scottish Prison Service (UK). The research process began with identifying a central point of contact, who signed a confidentiality agreement and assisted with recruitment. This individual was an affiliate of the United Kingdom prison system. We selected two prisons from the UK that were considered exemplar models based on their family-centric practices and programs. We also identified two charities whose staff worked in and provided services (e.g. reception check-in and working in visiting areas) at each of these prisons. We then used snowball sampling and chain referrals to recruit participants. Telephone and email were the main platforms for communication regarding logistics. After identifying potential participants, we forwarded data collection agreements, informed consent forms, interview guides, confidentiality agreements, and research fliers, collectively via email. Each respondent received the aforementioned research recruitment documents at least ten days before on-site interviews. Included in the informed consent was language stipulating that staff/employees were under no pressure to participate; we particularly emphasized the volunteer nature of this study. Our study began in October, 2016, for a 10-day duration.

\subsection{Site Locations}

Data collection and semi-structured interviews occurred at two prison facilities and two advocacy centers; home office of contract and volunteer staff who worked in the aforementioned prisons. Unlike many United States prisons, in the United Kingdom, advocacy and charity workers are often embedded as staff working in designated prison visiting areas and visiting centers.

Site officials provided the research team with private interview rooms. We also visited and observed additional prisons and advocacy centers to better understand United Kingdom prisons.

\subsection{Inclusion Criteria}

To qualify for this study, participants had to be over 18 years of age, speak fluent English, agree to informed consent, to be authorized and agree to take part in the study, and work, volunteer, or conduct research at a UK prison visit center or prison facility.

\subsection{Interview Protocol}

Twelve individual semi-structured face-to-face interviews and one telephone interview were conducted, with respondents, using conversational probes (see [28]). Each interview lasted approximately 60 to 90 minutes. Participants were provided a crisis resource list for assistance in case they experienced emotional discomfort during or after their involvement with the study. After participants granted permission, all interviews were audio-recorded. Observational data were recorded in the form of field notes describing how the staff members performed relevant tasks, especially their interactions with families who came to the prison visiting areas. According to Runeson and Host [28], this type of observation involves one-way engagement and represents a non-intrusive method of capturing the ways in which specific work is carried out by the individuals who make up the sample. We were granted special permission to photograph visiting areas with no occupants.

\subsection{Data Analysis}

Three researchers conducted cross-case analyses. This analysis served to compare similarities, and differences between the three cases, as well as identify emerging themes captured in a database created specifically for this study. To determine the completeness of each case, we examined: archival records; pamphlets; public service announcements; 
training manuals; photographs; public policy documents; interviews, and field notes. These data were reviewed until further analysis ceased to add new data relevant to address the research questions [25].

Multiple triangulation methods helped to decrease the likelihood of researcher bias and enabled the validation of assumptions and interpretations of the data. This work was carried out in a manner consistent with the triangulation methods proposed by Runeson and Host [28] to establish: (1) a clear chain of evidence documented through step-by-step processes, such as audit trails managed by the principal researcher; (2) flexible and parallel data analysis during data collection to enable the investigation of emergent insights, and (3) first- and second-order coding processes. By way of a final triangulation, multiple researchers reviewed, analyzed, and challenged the de-identified coded data. Initial codes generated by one researcher were subsequently compared to those that had been created by a second investigator. Preliminary codes were then captured in a table and summarized (see example Appendix A, Table 1). A third researcher performed a review of the coding and thematic content through a negative case analysis that challenged the initial themes and conclusions by offering rival explanations [28]. These data were then reconciled for reporting, as shown in the results below.

\section{Result}

This section presents the findings from the research. Where fitting, direct anonymized quotes have been used to illustrate key findings. In some cases, we provide photographs to help illustrate the results.

In this study, prison staff described their roles during the visiting processes as an evolution originating from traditional corrections-based practices (i.e. policing) to a visiting program capable of accommodating three goals: low-stress family visits, inmate conformance, and reduced recidivism. Each of these goals is described in the six themes that follow as aligned with each research question. In the first research question, we asked, RQ1. How do prison staff interpret their roles during family visiting experiences?

\subsection{Prison Staff Roles: Dichotomous Subculture | Bifurcated Identities}

To achieve a low-stress family visits program, some staff reported the tensions of balancing institutional controls with relaxed and less punitive roles of prison staff towards families. This further contributed to a dichotomous relationship phenomenon. In essence, prison staff carried out their primary roles as officers responsible for maintaining a controlled and secure custodial environment while also being supportive of children and families in visiting areas.

The two prisons used in this study were considered family-centric facilities with low stress visiting environments. Thus, at the outset of creating these environments prison staff hired employees whose skill sets - aligned with administering tailored programming for families. To accomplish these goals, prison staff integrated and paired workers with traditional corrections backgrounds to work alongside prison staff with prior experience in social services, advocacy, and human services. Prison staff in leadership roles expressed pride in creating programs that addressed the needs of families. There was a consensus among staff, from all cases, that they felt empowered to act independently and had the freedom to exercise moral judgment on how to treat visitors. One prison created an entire wing (prison tier or unit) dedicated to encouraging frequent and quality bonding experiences for inmates and their families. From a leadership perspective, the family visits program aligned with national and international policy initiatives (e.g. The United Nations Convention on the Rights of the Child). These programs were believed to yield financial and social returns on investment. A private sector staff member recalled,

"See, 10 years ago, there was so much research evidence about the importance of engaging family, maintaining family ties...so we started to package all that together and make a case....it's quite clear, the evidence is there from the central government itself and internally, correction services evidence is saying the same thing - healthy family contact is a cause or factor on reducing reoffending (recidivism)."

A staff member from the private prison also recommended strategies for a family-based visiting model. The respondent indicated that through the improvement of family programs and engagement, they (staff) could motivate inmate behavior.

"... when I was asked to look at the family program which I knew nothing about, it was at that point, it became a turning point for me. I guess you would say like an epiphany. I realized when prisoners were talking about their children and their families, they were more engaged - more motivated than when we talked about anything else. So, I started to think that if we could capture that motivation and apply that to a model, then you're taking the best bits of what works, engages, and motivates prisoners."

To work effectively in the prison visiting areas, respondents in the public prison group felt that to fulfill their roles efficiently, required specific training and backgrounds. A public sector prison staff member reflected on roles and training,

"I had a lot of training on the job so, I have [participated in] different behavior programs. You need to have the training to be able to deliver. I would consider the work I did as a prison officer as important if not more important than a lot of the intervention experience."

While the essence of family-centric visits is based on showing empathy, prison staff explained that, at times, they were torn between engendering displays of humane treatment versus demonstrating a traditional corrections-based persona. This created a dichotomous subculture where the corrections-based prison officer's bifurcated roles intersected with family-intervention. Prison staff indicated that their roles were unlike typical corrections-oriented positions (private and public sector prisons). In essence, their roles were redefined. This difference is captured in the recollection of a prison officer, from the public sector prison, who indicated, “... it 
certainly wouldn't be uncommon for a prison officer to be having a brew (tea) with a prisoner."

The transition was easier for advocacy staff members with social work backgrounds, and congruent with their training in the helping profession. Along the same lines, a private prison staff member recalled, "During the visits, the managers will monitor things. The security department will analyze intelligence and look for patterns. Additionally, we watch members of staff on CCTV every period of the day, checking for patterns." The manager-led surveillance enabled the staff to perform their roles with families without overtly having to monitor visitors.

\subsubsection{Prison Visit Programs as Interventions}

Perspectives held among private or public sector prisons and the advocacy groups were that continuity of family care was essential. This care entailed wraparound intervention, a priority supporting the well-being of the entire family. In each case, staff indicated as visiting programs emerged, the staff's interpersonal engagement and ties germane to working with families and their children made it difficult to have displays of a punitive, uncaring, or cold attitude towards inmates, as illustrated in one officer's reflection,

"You speak to someone on the wing and you got to discipline them because they've done something stupid, then an hour later, you're going to be sitting down, playing chess with him, as you would with a friend. "

\subsubsection{Evaluating Success}

Prison staff conveyed that it was important to evaluate how well they were succeeding with the family-based visiting programs. The level of services provided by visits staff varied among the two prisons/advocacy as reported through 3 cases. A respondent from the public sector prison shared that family services ranged from facilitating a financial travel stipend for family hardships to providing external therapeutic referrals. On the other hand, the consensus held among staff working at the private prisons conveyed that it was commonplace to contact a social worker when children were struggling in school. Advocacy staff articulated that it was imperative that staff provide children with on-site wraparound counseling services when the distress was observed before, during, and after visits. This continuity of intervention extended as an outreach to school counselors, interagency-partners, and other programs to help the child cope with separation from an incarcerated parent. Prison staff also made referrals to inter-agency organizations and community charities or nonprofits outside of the prison complex. This offered additional therapeutic support to families.

In the private and public prisons, the staff clarified that their role was aligned with engaging with families and creating a welcome atmosphere during prison visits. One respondent reflected,

"It was a warm, and a safe environment for people who came and waited to go into the prison for the visit... open space, soft, non-threatening... it (visiting room) looks... like... a living room or [what] American people call the lounge."

The advocacy workers/visits staff also saw their role as instrumental in offering psychosocial services to families. Examples of services offered at facilities included onsite programming and a call center function dedicated as a family helpline. For example, a staff member indicated,

"[We] are set up to assist any family member that is within the prison system and we can provide one-on-one support and help people see the benefits [of this support]... we can refer the families to a local charity and advocacy group that provides intervention."

For private and public prisons, the visits staff indicated that one important example of success entailed seeing children bonding and playing with each other. This is illustrated via private sector staff reflection,

"When mom's down the lounge, she's gets to speak to the other mums (mothers). And they -- really click. They are mums, they enjoy coming into the prison visiting areas on the first Monday of the month. "

\subsubsection{Our Role Is to Balance Safety and Security}

Public and private sector prison staff considered their roles congruent with effective operations during visits. In their roles, staff addressed misconduct and ensured the safety of all occupants in the designated visiting spaces. When reflecting on their approach to discipline or to address any security issues, there was little differentiation between private and public sector prisons. Specifically, the staff informed us that they used a de-escalation process that required them to be more thoughtful decision makers in the case of violations and misconduct. Alternatives to overt or public displays of discipline included waiting to address the inmate infraction after the family visit was over. Most of the staff (30\% of the total sample across the cases) considered it to be imperative to balance safety and security in a manner that allowed leverage to use traditional discipline, but not at the expense of traumatizing children or their families.

All prison staff across each case stressed the importance of using empathy in the discharge of their duties. A strong subtheme emerged in private prisons, which we entitled, "I am a prison officer, first." This theme suggests that, even within a family-focused environment, security remained the priority. Respondents from the advocacy group held firm that at all costs, children should be protected from trauma.

\subsection{Physical Space and Human Value}

Based on case respondents' comments, in their role, they considered the implementation of enhancements to physical environments as tangible evidence that staff cared about visitors' experiences in carceral spaces. We aptly named this theme: physical space equates to human value.

In varying ways, all staff members asserted that the visiting spaces showcased their humanity, especially their concern for children. In a research question, we asked: How do prison staff use spatial areas, including their form, size, scale, and color to represent family-visits spaces? [See 30]. Accordingly, the majority of the staff from all 3 cases (private, public, and advocacy) explained that the physical arrangement, furnishings, and coloring in visiting areas and play spaces 
were considered an outward expression of their empathy towards children and respect for family members who visited inmates. For the private prison, field notes and photographs reflected how each visiting area provided amenities and aesthetics for family members, especially children. While the architecture and design of some of the physical spaces included features that are unmistakably associated with prisons, such as bars and barbed wire, staff asserted that even modest upgrades and alterations (e.g., the addition of colored walls and increased lighting) served for prison staff to communicate their values as workers. This attitude was particularly evident when a public prison staff member contrasted the previous visiting settings with current renovations. The respondent noted,

"When you looked at the prison visiting area, at that time, clearly the programs were not working and wasn't anything to be proud of... a forgotten area of the prison - as prison visiting areas tends to be."

A non-managerial staff member from the advocacy group felt empowered to request the leadership at her facility to implement a family-based visiting program that included a facility with bright and colorful interior design and play areas for children. This resulted in prison staff enhancements of the interior and exterior. The prison extended the development to a recreational play space for children as illustrated in Figure 1.

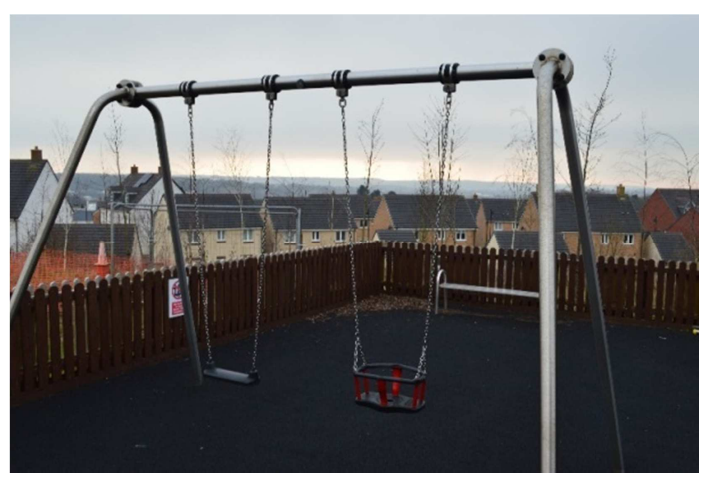

Figure 1. Playground on Prison Property. Background Community.

The majority of respondents across cases looked for ways to reward the good behavior of inmates during family visits, including creating a tiered level of comfort in the visiting seating areas. Based on merit, inmates without infractions were offered more comfortable seating for family visits. They were able to dine together, have tea, or sit together in a less constrained seating arrangement. Those who violated rules were not denied a visit, but rather sat in seating areas that offered the least comfort and fewer amenities. A private sector staff member recalled,

"...They got sofas in there, so it feels more like a family living room...the dads can actually pick up the children, put them on their knees. Everything is well thought out. Even the Zen Garden over there-I don't think there is anything that is put in this hall without having thought — careful thought behind it. And even these chairs here, this is for the enhanced area. "

The Zen Garden, shown in Figure 2, illustrates a picket fence as a boundary from the general prison visit area. The fish tank on the left and the tree adjacent to simulated rocks with embedded waterfall offers a tranquil background to the sofa seating.

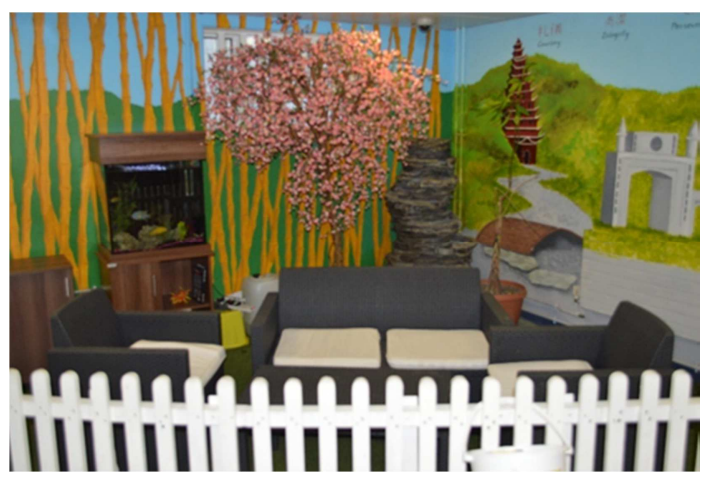

Figure 2. Zen Garden.

A staff member from the public prison stated, "It doesn't look like you're in prison... it looks like you are in a school hall or something like that. You know, you wouldn't think for a minute that all these people are inside the prison. And it's lively, the children are running around. But, I don't let my guard down ever."

Prison staff in the advocacy group considered the physical visiting center areas (adjacent to the prisons) to be vital; they provided inmates' families with space for multiple purposes: orientation, children's clothing exchange, and such amenities as food available for sale at a café.

Figure 3, illustrates an example of a prison in the UK with a visiting area. Here, a café, seating, counseling rooms, orientation, and a place to change clothes were provided.

\subsection{Shifts in Attitudes and Emergent Philosophies}

This last section addresses the research question regarding how prison staff perceived their attitudes and evolving roles towards family and during the visits. We asked, specifically: How do prison staff perceive the evolution of family-centric prison visiting environments?

There was a notable difference in beliefs held among all respondents regarding when the paradigm shift and evolution of the family-centric visiting model occurred at the prison. The prison visits staff indicated that the philosophy of having family-oriented visiting areas evolved over a period of time.

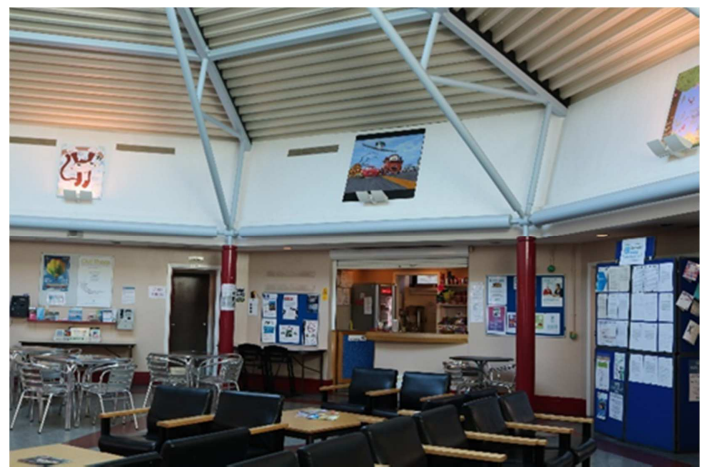

Figure 3. Example of What Visiting Areas with Café, Seating, And Orientation Spaces. 
Prison staff who had advocacy backgrounds believed that the attitudinal change towards families began when the leadership articulated the importance of humanizing the family to protect visiting children. In the public and private sector prisons, there was agreement among the management ranks that emphasis was placed on achieving favorable inmate behavior and reduced recidivism. These goals were an articulated priority and an impetus to the evolving visiting programs.

There was a consensus among cases that when the visiting conditions and associated environments improved, tensions between inmates and staff noticeably abated.

Finally, some private prison respondents indicated that the most significant shift in attitudes entailed moving from a punitive-oriented visiting process to one that was more sensitive to families. This change included implementing a model that included co-operations with advocacy workers who were trained in family interventions. In the cases that represented private- and -public sector prisons, most respondents noted shifts in attitudes when national policy mandated that prison staff focus on the well-being of children and families who were exposed to prison environments. These respondents posited that programs dependent on shared responsibilities among stakeholders (social workers, advocacy, and community members) reinforced family-focused philosophies and held each entity accountable.

\section{Discussion}

A significant number of public and private sector prisons in the UK have begun to recognize the role that prison staff can play in contributing to the health and psychological well-being of families and inmates in visiting settings. As researchers originating from the United States, where draconian visiting environments were reported as the norm [7], we set out to learn how prison staff in 2 United Kingdom prisons viewed their roles. The cases in this study represented perspectives from the private sector, public sector, and advocacy staff working in prison settings. Consistent with recent literature, staff who worked in the prison visiting settings found that positive interpersonal engagement towards family and children resulted in lower inmate aggression and contributed to their health and well-being [29].

In this study, staff extended services well beyond simply making the visit comfortable; to that which cultivated relationships with families. They ensured that safeguards to preserve familial contact were in place (such as rarely denying a visit under any circumstance, extending a clothing swap, and offering on-site counseling and wraparound services, and letting children be children in play areas). This phenomenon aligns with Bowen's [18] concepts of triangles, indicating when the family as a system is under stress, a third party can intervene to provide relief. In this study, prison visits environments became a form of intervention that took place in a centralized hub for social services offerings as well as physical comfort. Staff also saw their role as providing the capacity for families to build social ties in these environments that would ultimately contribute to family reunification after the inmate was released.

To assist family reunification, staff implemented merits-based visiting that came with special privileges. In some cases, the family-based prison visiting areas emulated home settings. Depending on the inmate's behavior, they could share a meal in a living-room-like setting. In other cases, visits were assigned seating. As photographs illustrated, bright, clean, and physically appealing visiting areas also meant that staff reaped the benefits of working in pleasant facilities rather than drab environments. Further discussion on space, environment, and configuration can be learned in a previous research paper about this study [30].

Prison staff perceived their roles and responsibility to entail maintaining structure, welcoming families, while also establishing boundaries that minimized the risk of visiting policy violations. The visiting center intervention and wrap-around services seemed to blur the role of traditional corrections-based prison staff. For instance, in some cases, staff roles were similar to that of a case manager or helping practitioners. Their responsibilities might include assessment and other programming to preserve intact family systems [31, $6,21]$. In these cases, the role of advocacy workers in either prison was a natural pairing because of their social work and helping professional backgrounds.

Our findings were counter to previous research where prison staff was reported as intimidating families during prison visits [7]. In general, some prisons institute penal philosophies underpinned by a rigid inflexible corrections-based retributive or utilitarian rationale, which collectively suggests that the inmates' violation of the law justifies the time spent in prison and the way they and their families are treated (punitive or kindly, respectively) [32]. A retributive philosophy rationalizes that society has the right to punish an offender and do so at an appropriate level [15]. Utilitarian philosophy holds that society is justified in its treatment of prisoners so long as it protects the public from harm [33]. One consequence of these philosophies of punishment is the denial of or restrictive prison visits [3].

In contrast, we found that UK prison management emphasis was placed on rehabilitation [34]. In this regard, when prison staff bonded with a prisoner's child, they found that inmates were motivated to demonstrate good behaviors.

While previous literature suggested that family engagement in prison settings can result in conflict or abrasiveness between prison staff and visitors [3], our findings suggest that prison staff can leverage their power in the form of benevolence and encourage good behavior through merit-based motivation. Thus, as a result, they created environments conducive to fostering the well-being of visiting families and saw a decline in inmate aggression. This in contrast to prison staff projecting their personal feelings of stress or frustration on others and the inconsistent implementation of practices, leaving families confused and exasperated [35]. This finding also challenges 
the conditions identified by Boudin et al. [3] in United States prisons, where staff have been found to be rigid and sometimes abrasive towards families. This is an important finding, given that projecting feelings, otherwise known as societal regression [18], can be harming to visitors. In the same way a parent can project their feelings on a child when they are under stress or frustrated, societal institutions also by way of their cultural and institutionalized behaviors can project frustration, controlling behaviors, and aggression onto the family unit.

In general, prison internal documentation and policies are not easy to acquire from UK public- or -private sector institutions, given that these documents may govern prison security operations. However, there are public archival documents that contribute to insights on the evolution of family-centric practices and their possible influence on staff roles, as mentioned by staff in our research. For instance, as a 25-year reflection, Day and colleagues [36], suggested that the prison reform movement began in the 1990s, as detailed in The Lord Woolf Report. This report covers the Strangeways riots as the landmark event that perpetuated change [37] in the United Kingdom offender management programs. The Woolf report documents prior draconian, unfair treatment of both inmates and their families. The aforementioned deplorable conditions [36] of United Kingdom prisons and ensuing riots resulted in a paradigm shift that changed the landscape for prison visits and inmate treatment.

In reflection, the humane treatment of families might be explained by the close pairing of helping professionals with workers who had traditional corrections backgrounds. This pairing may have created an accountability structure for family visits in prisons. Additionally, prison leadership adopted tenets from such international national policy as the UN Convention on the Rights of the Child [38, 39]. Specifically, international policy and human rights initiatives required institutions to above all else ensure the humane treatment and well-being of vulnerable children.

\section{Future Studies}

Within the scope of a single article, it was not possible to relate the vast detail and rich findings associated with this case study in its entirety. Future publications will provide further insights. In light of the stated findings in this report, subsequent research should examine the phenomenon of dichotomous subcultures. To accomplish this, a study could be designed to examine programs that offer familial intervention and consider what happens when families and children leave these safe havens (prisons that offer social and human services). It is unclear whether there is a continuity of care after the inmate returns home to their family. We recommend that future research examine how public and private prisons in the United States might develop family-centric visitation programs as pilot cases. Finally, future studies could extend our research focus and examine prison staff roles to standardize best practices.

\section{Limitations}

In our study, we used a small qualitative sample for analysis. While case studies generally comprise relatively small sample sizes, we recognize that our findings should, nevertheless, not be overstated or considered generalizable to all prisons in the UK. Additionally, cultural differences may need to be taken into consideration when applying these findings to prison visit settings in the United States. We also recognize that the models that we viewed were considered exemplary and that though they were deemed to have promising aspects, they may certainly have their challenges. Finally, we examined prison visiting settings as part of a planned research study and, accordingly, witnessed staff overseeing family interactions under conditions that may have been rendered in the best possible light.

\section{Conclusion}

There is established and notable evidence that family-centric programs at prisons can improve the morale of inmates. This paper adds to the literature on the benefits associated with improved family relations and inmate conformance when prison staff is emboldened by leadership to adopt pro-family interventions. Our observations and findings support previous assertions that prison staff also benefit when tensions were reduced between themselves, inmates, and visiting families. This state of calm made their role as officers in a prison less stressful.

We believe it is possible that prison institutions in the United States and other countries could implement merit-based privileges with family-centered visits to affect the attitudes and well-being of family, inmates, and staff. For example, enhanced visits could be associated with longer or more frequent visits, with less staff overhead. These upgrades could be based on an inmate's achievement in training, certificates, or other forms of positive behaviors.

Finally, prison staff may have been influenced by advocacy workers who were trained in social work and intervention as a motivator for their own treatment of families. It may be beneficial to integrate nontraditional criminal justice expertise, e.g., behavioral services, in prisons where administrators are endeavoring institutional change. Arditti [41] specifically discussed the importance of integrating prison staff with social workers and other stakeholders, which can serve as protective factors for visiting children and families Additional discussion is explained in greater detail in select publications [30, 42]. 


\section{Appendix}

Table 1. Sample Coding.

\begin{tabular}{|c|c|c|c|}
\hline Quote & First Order Code & Second Order Code & Major Theme \\
\hline $\begin{array}{l}\text { "Family crisis support is essential." } \\
\text { "We look for signs of contraband and } \\
\text { breaking rules, but not in an obvious manner" }\end{array}$ & $\begin{array}{l}\text { Wraparound Supports } \\
\text { Institute Covert Security Measures }\end{array}$ & $\begin{array}{l}\text { Security role with tensions of } \\
\text { providing intervention }\end{array}$ & Dichotomous Subculture \\
\hline $\begin{array}{l}\text { "There are creative ways to punish inmates" } \\
\text { "Create a family-friendly atmosphere" }\end{array}$ & $\begin{array}{l}\text { Humane Treatment } \\
\text { Support the parent and help the child }\end{array}$ & $\begin{array}{l}\text { "I am a Prison Officer First" } \\
\text { Prevent family trauma }\end{array}$ & $\begin{array}{l}\text { We Balance Safety \& } \\
\text { Security }\end{array}$ \\
\hline
\end{tabular}

Table 1 Example of Coding. This table contains a small segment of first- and -second order coding.

In Table 1, the initial first- and -second order coding illustrates a progression to major themes. In our preliminary findings reported in (Johnson, Johnson, \& Tate, 2018), we found that the major themes could be clarified by adding meaningful labels that aligned with the minor and major codes For example, initially, the code "We Balance Safety and Security" to "We Balance Safety \& Security with Empathy." Some other works have supported the idea of refining preliminary results $[6,16,25]$.

\section{Acknowledgements}

We acknowledge Barbara Benoliel, fellow researcher who provided insights and support to this research study. Additionally, we thank the United Kingdom, Her Majesty's Prison Service (HMPS), the Scottish Prison Service, study participants and our U.K. central point of contact for helping to make this study possible.

\section{References}

[1] Blagden, N.; Winder, B. \& Hames, C. (2016). "They treat us like human beings"-_Experiencing a therapeutic sex offender prison: Impact on prisoners and staff and implications for treatment. International Journal of Offender Therapy and Comparative Criminology, 60 (4), 371-396.

[2] Drake, D., Woodall, J. (2012). The significance of "the visit" in an English category B prison: Views from prisoners, prisoners' families and prison staff. Community Work and Family, 15, $29-47$

[3] Boudin C Stutz T Littman A 2013 A. Prison Visitation Policies: A Fifty-State Survey. Boudin, C.; Stutz, T. \& Littman, A. (2013). Prison visitation policies: A fifty-state survey. Yale Policy Review, 32 (1), 149-189. 201609100515031885050297.

[4] Bosworth, M., Parmar, A., \& Vázquez, Y. (Eds.). (2018). Race, criminal justice, and migration control: Enforcing the boundaries of belonging. Oxford University Press.

[5] Arditti, J. (2016). A family stress-proximal process model for understanding the effects of parental incarceration on children and their families. Couples and Family Psychology: Research and Practice, 5 (2), 65-88.

[6] Brutus, Liz (2011). Prisoners' families: The value of family support work. Families Outside, www.familiesoutside.org.uk [Accessed: Month Day, Year].

[7] Dixey, Rachel \& Woodall, James (2012). The significance of "the visit" in an English category B prison: Views from prisoners, prisoners' families and prison staff. Community Work and Family, 15, 29-47.

[8] Ismail, N., \& De Viggiani, N. (2018). Should we use a direct regulation to implement the Healthy Prisons Agenda in England? A qualitative study among prison key policy makers. Journal of Public Health, 40 (3), 598-605.

[9] Steiner, B., \& Wooldredge, J. (2018). Prison officer legitimacy, their exercise of power, and inmate rule breaking. Criminology, 56 (4), 750-779.

[10] De Claire, K., \& Dixon, L. (2017). The effects of prison visits from family members on prisoners' well-being, prison rule breaking, and recidivism: A review of research since 1991. Trauma, Violence, \& Abuse, 18 (2), 185-199.

[11] Hayes, D., Butler, M., Devaney, J., \& Percy, A. (2018). Allowing imprisoned fathers to parent: Maximising the potential benefits of prison-based parenting programmes. Child Care in Practice, 24 (2), 181-197.

[12] Burford, Gale (2017). Family group conferencing: New directions in community-centered child and family practice. New York, NY: Routledge.

[13] Crew, Ben; Liebling, Alison \& Hulley, S. (2015). Staff-prisoner relationships, staff professionalism, and the use of authority in public- and private sector prisons. Law \& Social Inquiry, 40 (1), 309-344.

[14] Hulley, S., Crewe, B., \& Wright, S. (2016). Re-examining the problems of long-term imprisonment. British Journal of Criminology, 56 (4), 769-792.

[15] Strang, Heather. \& Braithwaite, John. (2017). Restorative Justice: Philosophy to practice. New York: NY: Ashgate Publishing.

[16] Brooke, J., \& Jackson, D. (2019). An exploration of the support provided by prison staff, education, health and social care professionals, and prisoners for prisoners with dementia. The Journal of Forensic Psychiatry \& Psychology, 30 (5), 807-823.

[17] Kaakinen, J. R., Coehlo, D. P., Steele, R., \& Robinson, M. (2018). Family health care nursing: Theory, practice, and research. FA Davis.

[18] Bowen M 1978 Family therapy in clinical practice. Bowen, M. (1978). Family therapy in clinical practice. New Skipton, NY: Aronson.

[19] Foster, R. (2017). Exploring 'betwixt and between 'in a prison visitors' centre and beyond. In Carceral Spatiality (pp. 169-198). Palgrave Macmillan, London.

[20] Souryal, S. (2009). Deterring corruption by prison personnel. The Prison Journal, 89 (1), 21-45. doi. $10.1177 / 0032885508329979$ Sour. 
[21] Cochran, J. C.; Mears, Daniel P.; Bales, W. D. \& Stewart, E. A. (2013). Does inmate behavior affect post-release offending? Investigating the misconduct-recidivism relationship among youth and adults. Justice Quarterly, 53 (2), 220-254.

[22] Sudom, K., Dursun, S., Flemming, S. (2006). PERSTEMPO in the Canadian Forces: The Role of Coping and Cohesion in the Relationship between Job Stress and Morale. Retrieved from http://www.dtic.mil/dtic/tr/fulltext/u2/a472680.pdf.

[23] Brunton-Smith, I. \& McCarthy, D. J. (2017). The effects of prisoner attachment to family on re-entry outcomes: A longitudinal assessment. The British Journal of Criminology, 57 (2), 463-482.

[24] Woodall, J., \& Kinsella, K. (2018). Striving for a "good" family visit: The facilitative role of a prison visitors' centre. Journal of Criminal Psychology.

[25] Yin R 2009 Case study research: Design and methodsYin, Robert, K. (2009). Case study research: Design and methods (4th ed.). Thousand Oaks, CA: Sage Publications, Inc.

[26] Ponelis, S. (2015). Using interpretive qualitative case studies for exploratory research in doctoral studies: A case of information systems research in small and medium enterprises. International Journal of Doctoral Studies. 10 (2015), 534-550.

[27] Leitch, C. M., Hill, F. M., \& Harrison, R. T. (2010). The philosophy and practice of interpretivist research in entrepreneurship: Quality, validation, and trust. Organizational Research Methods, 13 (1), 67-84.

[28] Runeson, P., \& Host, M. (2009). Guidelines for conducting and reporting case study research in software engineering. Empirical Software Engineering. 14 (1), 131-164. doi: 10.1007/s10664-008-9102-8.

[29] Clancy, A., \& M., M. (2017). Prisoners and their children: An innovative model of 'whole family'support. European Journal of Probation, 9 (3), 210-230.

[30] Hart-Johnson, A., Johnson, G., \& Tate, M. (2018). Prison staff who shape child and family visits: United Kingdom multiple case study. In Gordon, L. (Ed), Contemporary Research and Analysis on the Children of Prisoners: Invisible Children. pp 241-265. Cambridge Scholars Publishing.

[31] Arditti, J. \& Jyoti S. (2015). Parental incarceration and child trauma symptoms in single caregiver homes. Journal of Child and Family Studies, 24 (3), 551-561.
[32] Lerman, A. E. \& Page, J. (2012). The state of the job: An embedded work role perspective on prison officer attitudes. Punishment \& Society. 14 (5), 503-529. doi: $10.1177 / 1462474512464135$.

[33] Bosworth, M. (2017). Engendering resistance: Agency and power in women's prisons. New York, NY: Routledge.

[34] HM Prison \& Probation. (2019). HMPPS business strategy: Shaping our future. Retrieved from: http://www.govwire.co.uk/news/national-probation-service/gu idance-hmpps-business-strategy-shaping-our-future-34635.

[35] Shannon, S., \& Page, J.(2014). Bureaucrats on the cell block: Prison officer's perception of work environment and attitudes towards prisoners. Social Services Review, 88 (4), 630-657. doi: https://doi.org/101086/678448.

[36] Day, M., Hewson, A., \& Spiropoulos, C. (2015). Strangeways 25 Years On. London: Prison Reform.

[37] Prison Reform Trust. (1991). The Woolf report: A summary of the main findings and the recommendations of the inquiry into prison disturbances. Retrieved from www.prisonreformtrust.org.uk/Portals/0/Documents/Woolf $\% 2$ Oreport.pdf.

[38] UNICEF (n. d.) United Nations Convention on the Rights of the Child. Retrieved from https://www.unicef.org/rightsite/237.htm.

[39] United Nations Human Rights Office of the High Commissioner. (1990). Universal periodic review - United Kingdom. Retrieved from https://www.ohchr.org/en/professionalinterest/pages/crc.aspx.

[40] Goffman, E. (1961). On the characteristics of total institutions. In Symposium on preventive and social psychiatry (pp. 43-84). Washington, DC: Walter Reed Army Medical Centre.

[41] Arditti, J. (2004). Families and incarceration: An ecological approach, http://www.convictcriminology.org/pdf/arditti/e-FamiliesIncar ceration.pdf [Accessed: March 30, 2018].

[42] Hart-Johnson, A., Benoliel, \& B., Johnson, G. (2018). Photographic Data as Content for Analysis in Qualitative Research. In Garris, B. \& Waters, A. Eds), Conference Proceedings 2018 NOHS National Conference Philadelphia, PA. pp 79-82. National Organization of Human Services. 